\title{
Michael G. Stewart and Samuel H. Selesnick (eds): Differential diagnosis in Otolaryngology: head and neck surgery
}

\author{
Published by Thieme Verlag, 2011, ISBN 978-1-604-06051-5, p 498
}

\author{
Klaus Albegger
}

Received: 15 May 2011/ Accepted: 16 May 2011/Published online: 25 June 2011

(C) Springer-Verlag 2011

This relatively small book is a very practical resource for rapid and accurate diagnosis and differential diagnosis in Otorhinolaryngology-head and neck (including facial plastic) surgery. This comprehensive manual uses an innovative format what ORL-specialists need in their daily practice.

Each chapter is symptom based, with the patient's presentation followed by an easily accessible list of potential diagnoses - the differential diagnosis. Supplementary data on the features of different diseases help the user to correctly identify the underlying problem.

As the main chapters are organized by signs and symptoms and not by disease, this book becomes a ready clinical reference. With the key information, one can then assess a variety of complaints. The diagnostic evaluations discussed include all classical methods as well as new and forthcoming technologies in Allergy and Immunology, Genetics, Audiology [auditory steady state responses (ASSR)] and vestibular testing [vestibular-evoked myogenic potentials (VEMPs)], MRI and PET/CT.

In detail, the authors present symptom-orientated evaluations in Neurotology, Rhinology, Upper Aerodigestive Tract, Voice, Facial Plastic and Reconstructive Surgery, as well as Allergy and Immunology, with special focus on head and neck imaging and positron emission tomography.
The differential diagnosis (DD) in Neurotology, Rhinology/sinus disease, skin/face and neck disorders are separated for adult and pediatric patients, whereas the DD for oral cavity, larynx/trachea, pharynx/esophagus include adults and children. There is also a chapter on DD in cosmetic and plastic surgery, trauma and reconstruction. The book closes with future prospects in new technologies for DD.

Numerous cross references throughout the text clearly link different symptoms and diseases to provide a solid basic understanding of each diagnosis. Eighty-two highquality illustrations and clinical photographs, including 52 in full color, clearly demonstrate many lesions and diseases. Well-organized tables of rare diseases and syndromes enhance a rapid overview.

DD in Otolaryngology is a "missing link" in the ORLHNS literature. It is a recommended reference and refresher for experienced ORL-HNS specialists, who need to update their knowledge of their own and other subspecialty fields in ORL-HNS. But it is also a must-have for ORL residents and a very valuable tool for younger fellows when they prepare for daily practice or study for exams.

K. Albegger ( ()

Paracelsus Medical University, Salzburg, Austria

e-mail: klaus.albegger@pmu.ac.at 